\title{
Stochastic Operators, Information, and Entropy
}

\author{
Jürgen Voigt \\ Mathematisches Institut der Universität München, D-8000 München 2, \\ Federal Republic of Germany
}

\begin{abstract}
For a stochastic operator $U$ on an $L_{1}$-space, i.e. $U$ is linear, positive, and norm preserving on the positive cone of $L_{1}$, it is shown that $U$ decreases relative information between two nonnegative $L_{1}$-functions. Furthermore it is shown that the following properties of $U$ are closely related: $U$ is energy decreasing (energy preserving), $U$ is $H$-decreasing, where $H$ is Boltzmann's $H$-functional, and the Maxwell distributions are fixed points of $U$.
\end{abstract}

The aim of this note is to prove some properties of stochastic operators on $L_{1}$ spaces. In Sect. 1 we show that a stochastic operator decreases relative information between two nonnegative $L_{1}$-functions. Such a property was known for special cases.

In Sect. 2 we show that, for a stochastic operator $U$, certain properties are equivalent. If $\alpha$ is a function on the measure space defining the energy and $H$ is Boltzmann's $H$-functional, then, for instance, it is shown that $U$ is energy decreasing and $H$-decreasing if and only if all "Maxwell distributions" $\exp (-\kappa \alpha)(\kappa \geqq 1)$ are invariant under $U$. These properties are also equivalent to the property that $U$ is energy preserving and leaves one "Maxwell distribution" $\exp (-\alpha)$ fixed.

In [13], the author proves the $H$-theorem for Boltzmann type equations $u^{\prime}$ $=T u+J(u)$ in $L_{1}(\mu)$, for some measure space $(\Omega, \mathscr{A}, \mu)$. The required conditions are posed in abstract form on the strongly continuous semigroup $(U(t) ; t \geqq 0)$ of "free motion" generated by $T$, and on the "collision operator" $J$ separately. In applications, $U(t)$ should be expected to be a stochastic operator for each $t \geqq 0$. As a consequence of Theorem 2.1 and Proposition 2.5, one can obtain relations between some of the conditions for $(U(t))$; this is discussed in [13, remarks preceding Proposition 3.1]. As an example we consider $\Omega=D \times \mathbb{R}^{3}$, where $D \subset \mathbb{R}^{3}$ is open (and has suitable boundary), $\mu$ is Lebesgue measure, and $T$ is an operator associated with the differential expression $-\xi \cdot \operatorname{grad}_{x}$ and a 
suitable boundary condition. The corresponding initial boundary value problem in $L_{1}(\mu)$ is treated in [14]. With $\alpha$ defined by $\alpha(x, \xi):=1+|\xi|^{2}(x \in D$, $\left.\xi \in \mathbb{R}^{3}\right)$, the problem to find boundary conditions such that the corresponding semigroup satisfies the conditions required in [13] is discussed in [14, Sect. 9]. In this discussion, the equivalence of the conditions in Theorem 2.1 suggests that only rather restricted boundary conditions come into question.

The author is indebted to H. Spohn for suggesting Theorem 1.1.

\section{A Convexity Theorem}

Let $(\Omega, \mathscr{A}, \mu)$ be a measure space. By $L_{1}(\mu)$ we denote the space of real valued integrable functions, by $L_{1}(\mu)_{+}:=\left\{f \in L_{1}(\mu) ; f \geqq 0\right\}$ its positive cone. For $f, g \in L_{1}(\mu)_{+}$, we define the information of $f$ with respect to $g$ by

$$
H(f \mid g):=\int(f \ln f-f \ln g) d \mu .
$$

(We set $0 \ln 0=0, x \ln 0=-\infty$ for $x>0$.) For the introduction of this quantity we refer to [5]. The following remarks recall that we always have $-\infty<H(f \mid g) \leqq \infty$. The elementary inequality

$$
x \ln x-x \geqq x \ln y-y
$$

$(x, y \geqq 0)$ implies $f(w) \ln f(w)-f(w) \ln g(w)-f(w)+g(w) \geqq 0 \quad(w \in \Omega), H(f \mid g) \geqq\|f\|$ $-\|g\|>-\infty$. If in particular $\|f\|=\|g\|$, then $H(f \mid g) \geqq 0$. Furthermore, since equality in (1.2) holds if and only if $x=y$, we obtain $H(f \mid g)=\|f\|-\|g\|$ if and only if $f=g$.

Let $\left(\Omega_{i}, \mathscr{A}_{i}, \mu_{i}\right), i=1,2$, be measure spaces, and $U: L_{1}\left(\mu_{1}\right) \rightarrow L_{1}\left(\mu_{2}\right)$ a linear operator. $U$ is called positive if $U\left(L_{1}\left(\mu_{1}\right)_{+}\right) \subset L_{1}\left(\mu_{2}\right)_{+}$. (This implies that $U$ is continuous; cf. [11, II, Theorem 5.3, p. 84].) $U$ is called stochastic if $U$ is positive and $\|U f\|=\|f\|$ holds for all $f \in L_{1}\left(\mu_{1}\right)_{+}$(cf. [11, III, Def. 8.8, p. 191]; in $[4$, Def. 11.7 .4$, p. 353], such operators are called transition operators). We note that, for a stochastic operator $U$, we have $\int U f d \mu_{2}=\left\|U f^{+}\right\|-\left\|U f^{-}\right\|$ $=\left\|f^{+}\right\|-\left\|f^{-}\right\|=\int f d \mu_{1}$ for all $f \in L_{1}\left(\mu_{1}\right)$.

1.1. Theorem. Let $\left(\Omega_{i}, \mathscr{A}_{i}, \mu_{i}\right), i=1,2$, be measure spaces. Let $U: L_{1}\left(\mu_{1}\right) \rightarrow L_{1}\left(\mu_{2}\right)$ be a stochastic operator. Then

$$
H(U f \mid U g) \leqq H(f \mid g)
$$

holds for all $f, g \in L_{1}\left(\mu_{1}\right)_{+}$.

1.2. Remark. A statement similar to Theorem 1.1, for completely positive tracepreserving maps on the trace class operators on a Hilbert space, can be found in [6]; cf. also [12]. For special situations, Theorem 1.1 is known. We refer to [5, Chap. 2, Theorem 4.1, p. 19] for the case that $U$ is the operator forming conditional expectations, and to [7, Sect. 2.3], where our Corollary 1.5 is proved if $(\Omega, \mathscr{A}, \mu)$ is a discrete finite measure space. 
1.3. Lemma. Let $(\Omega, \mathscr{A}, \mu)$ be a measure space. Then there exists a compact Hausdorff space $S$ and an isometric algebra isomorphism $J: L_{\infty}(\mu) \rightarrow C(S)\left(L_{\infty}(\mu)\right.$ and $C(S)$ real valued). If $I=\bar{I} \subset \mathbb{R}$, and $\gamma: I \rightarrow \mathbb{R}$ is continuous, and $f \in L_{\infty}(\mu)$ is such that $\mu(\{w \in \Omega ; f(w) \notin I\})=0$, then

$$
J(\gamma \circ f)=\gamma \circ(J f) .
$$

Proof. Since the complex valued $L_{\infty}(\mu ; \mathbb{C})$ is a commutative $B^{*}$-algebra with unit (cf. [4, Definition 1.15.3, p. 22]), there exists a compact Hausdorff space $S$, and an isometric $B^{*}$-algebra isomorphism $\tilde{J}: L_{\infty}(\mu ; \mathbb{C}) \rightarrow C(S ; \mathbb{C}$ ) (cf. [4, Theorem 4.22.1, p. 157]). Then $J:=\tilde{J} \mid L_{\infty}(\mu)$ has the asserted properties.

If $\lambda \in \mathbb{R} \backslash I$, then $\lambda 1-f$ is invertible in $L_{\infty}(\mu)$, therefore $\lambda 1-J f$ is invertible in $C(S)$, and this implies $J f(s) \neq \lambda$ for all $s \in S$. Without restriction we may assume that $I$ is compact. Then there exists a sequence of polynomials $\left(p_{k}\right.$; $k \in \mathbb{N})$ such that $\max \left\{\left|p_{k}(t)-\gamma(t)\right| ; t \in I\right\} \rightarrow 0(k \rightarrow \infty)$. Now, $p_{k} \circ f \rightarrow \gamma \circ f$ in $L_{\infty}(\mu)$, $p_{k} \circ J f \rightarrow \gamma \circ J f$, and $J\left(p_{k} \circ f\right)=p_{k} \circ(J f)(k \in \mathbb{N})$ imply (1.4).

For the validity of (1.4) in a related situation we refer to [3, Theorem 4.6.18, p. 274].

1.4. Lemma. Let $S_{1}, S_{2}$ be compact Hausdorff spaces. Let $R: C\left(S_{1}\right) \rightarrow C\left(S_{2}\right)$ be a positive linear operator, $R 1=1$. Let $I=\bar{I} \subset \mathbb{R}$ be an interval, $\gamma: I \rightarrow \mathbb{R}$ continuous and convex. If $\varphi \in C\left(S_{1}\right)$ is such that $\varphi\left(S_{1}\right) \subset I$, then $R \varphi\left(S_{2}\right) \subset I$, and

$$
\gamma \circ(R \varphi) \leqq R(\gamma \circ \varphi) \text {. }
$$

Proof. Let $s \in S_{2}$. Then a positive linear functional $R_{s}$ on $C\left(S_{1}\right)$ is defined by $R_{s} \varphi:=(R \varphi)(s) \quad\left(\varphi \in C\left(S_{1}\right)\right)$, and, by the Riesz representation theorem, there exists a positive Borel measure $\mu_{s}$ on $S_{1}$ such that $\int \varphi d \mu_{s}=R_{s} \varphi\left(\varphi \in C\left(S_{1}\right)\right)$ (cf. [10, Theorem 2.14, p. 40]). By $R_{s}(1)=1$, we have $\mu_{s}\left(S_{1}\right)=1$. Jensen's inequality (cf. [10, Theorem 3.3, p. 61]) now implies $\gamma\left(\int \varphi d \mu_{s}\right) \leqq \int(\gamma \circ \varphi) d \mu_{s}$, which can be written as $\gamma(R \varphi(s)) \leqq R(\gamma \circ \varphi)(s)$. Since this is true for all $s \in S_{2}$, we have (1.5).

Proof of Theorem 1.1. We define the function $\gamma:[0, \infty) \rightarrow \mathbb{R}$ by $\gamma(t):=t \ln t$ (recall $0 \ln 0=0$ ). $\gamma$ is continuous and convex. Furthermore we note that, for $t_{1} \geqq 0$, $t_{2}>0$, we have

$$
t_{2} \gamma\left(\frac{t_{1}}{t_{2}}\right)=t_{1} \ln t_{1}-t_{1} \ln t_{2} .
$$

(i) In the first step we are going to prove (1.3) under the additional assumption that there is $c>0$ such that $f \leqq c g$. Let $g \in L_{1}\left(\mu_{1}\right)_{+}$be fixed, and define $\Omega_{2}^{\prime}:=\left\{w_{2} \in \Omega_{2} ; U g\left(w_{2}\right) \neq 0\right\}$. We define a linear operator $R^{\prime}: L_{\infty}\left(\mu_{1}\right)$ $\rightarrow L_{\infty}\left(\mu_{2} \mid \Omega_{2}^{\prime}\right)$ by

$$
R^{\prime} \varphi:=\frac{1}{U g} U(\varphi g)
$$

Then we have $R^{\prime} \varphi \geqq 0$ for $\varphi \geqq 0, \quad R^{\prime} 1=1$. Let $J_{1}: L_{\infty}\left(\mu_{1}\right) \rightarrow C\left(S_{1}\right), \quad J_{2}$ : $L_{\infty}\left(\mu_{2} \mid \Omega_{2}^{\prime}\right) \rightarrow C\left(S_{2}\right)$ be the algebra isomorphisms whose existence was shown in Lemma 1.3. Then the operator $R:=J_{2} R^{\prime} J_{1}^{-1}$ obviously has the properties 
required in Lemma 1.4 .

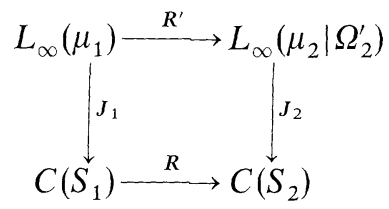

For $\varphi \in L_{\infty}\left(\mu_{1}\right)_{+}$we have $J_{1} \varphi \geqq 0$, and Lemma 1.4 implies $\gamma \circ\left(R J_{1} \varphi\right)$ $\leqq R\left(\gamma \circ J_{1} \varphi\right)$, or $\gamma \circ\left(J_{2} R^{\prime} \varphi\right) \leqq J_{2} R^{\prime} J_{1}^{-1}\left(\gamma \circ J_{1} \varphi\right)$. Applying $J_{2}^{-1}$ and using (1.4), we obtain $\gamma \circ\left(R^{\prime} \varphi\right) \leqq R^{\prime}(\gamma \circ \varphi)$,

$$
\begin{aligned}
\gamma \circ\left(\frac{1}{U g} U(\varphi g)\right) & \leqq \frac{1}{U g} U((\gamma \circ \varphi) g), \\
(U g)\left(\gamma \circ\left(\frac{1}{U g} U(\varphi g)\right)\right) & \leqq U((\gamma \circ \varphi) g) .
\end{aligned}
$$

Now let $f \in L_{1}\left(\mu_{1}\right)_{+}$be such that there exists $c>0$ such that $f \leqq c g$. Then there exists $\varphi \in L_{\infty}\left(\mu_{1}\right)_{+}$such that $f=\varphi g$, and for each $\varphi$ with these properties we have $(\gamma \circ \varphi) g=f \ln f-f \ln g$. [This is trivial for those $w_{1} \in \Omega_{1}$ for which $g\left(w_{1}\right)=0$ holds; otherwise it follows from (1.6).] From (1.7) we therefore obtain

$$
U f \ln U f-U f \ln U g \leqq U(f \ln f-f \ln g)
$$

on $\Omega_{2}^{\prime}$, where we have transformed the expression resulting on the left hand side with the aid of (1.6). From $f \ln f-f \ln g=(\gamma \circ \varphi) g$ we obtain $\mid f \ln f$ $-f \ln g \mid \leqq\|\gamma \circ \varphi\|_{\infty} g$, which implies $U(f \ln f-f \ln g) \mid \Omega_{2} \backslash \Omega_{2}^{\prime}=0$. Since we also have $U f\left|\Omega_{2} \backslash \Omega_{2}^{\prime}=0=U g\right| \Omega_{2} \backslash \Omega_{2}^{\prime}$, we obtain (1.8) on all of $\Omega_{2}$. Now, integrating (1.8), we obtain

$$
H(U f \mid U g) \leqq \int_{\Omega_{2}} U(f \ln f-f \ln g) d \mu_{2}=H(f \mid g) .
$$

(ii) In this step we assume $f \mid[g=0]=0 \mu_{1}$-almost everywhere. Defining $f_{k}:=f \wedge k g(k \in \mathbb{N})$, we have $f_{k} \leqq f_{k+1}(k \in \mathbb{N}), f_{k} \rightarrow f$ in $L_{1}\left(\mu_{1}\right)$, and from part (i) we obtain

$$
H\left(U f_{k} \mid U g\right) \leqq H\left(f_{k} \mid g\right) .
$$

If $H(f \mid g)=\infty$, then (1.3) is trivially true. Assume now $H(f \mid g)<\infty$. We define $\Omega_{1}^{\prime}:=[f \leqq g], \Omega_{1}^{\prime \prime}:=[f>g]$. Then we have $\left(f_{k} \ln f_{k}-f_{k} \ln g\right) \mid \Omega_{1}^{\prime}=(f \ln f$ $-f \ln g) \mid \Omega_{1}^{\prime}(k \in \mathbb{N})$. For $w \in \Omega_{1}^{\prime \prime}$ we have

$$
0 \leqq f_{k}(w) \ln f_{k}(w)-f_{k}(w) \ln g(w)=f_{k}(w) \ln \frac{f_{k}(w)}{g(w)} \nearrow f(w) \ln \frac{f(w)}{g(w)} \quad(k \rightarrow \infty) .
$$

The dominated convergence theorem therefore implies

$$
H(f \mid g)=\lim H\left(f_{k} \mid g\right) .
$$


As an increasing sequence, the sequence $\left(U f_{k}\right)$ converges to $U f \mu_{2}$-almost everywhere. From (1.2) we obtain $U f_{k} \ln U f_{k}-U f_{k} \ln U g-U f_{k}+U g \geqq 0$, and therefore Fatou's lemma implies

$$
\begin{gathered}
H(U f \mid U g)-\|U f\|+\|U g\| \leqq \liminf \left(H\left(U f_{k} \mid U g\right)-\left\|U f_{k}\right\|+\|U g\|\right), \\
H(U f \mid U g) \leqq \liminf H\left(U f_{k} \mid U g\right) .
\end{gathered}
$$

From (1.9), (1.10), (1.11) we obtain (1.3).

(iii) If the assumption made in (ii) is not satisfied, i.e. if $\mu_{1}\left(\left\{w \in \Omega_{1} ; g(w)=0\right.\right.$, $f(w) \neq 0\})>0$, then $H(f \mid g)=\infty$, from the definition, and (1.3) is trivially satisfied.

Theorem 1.1 becomes especially interesting if the "reference quantity" $g$ is a fixed point of $U$.

1.5. Corollary. Let $(\Omega, \mathscr{A}, \mu)$ be a measure space. Let $U: L_{1}(\mu) \rightarrow L_{1}(\mu)$ be a stochastic operator. Let $g \in L_{1}(\mu)_{+}$be a fixed point of $U$ (i.e. $U g=g$ ). Then, for all $f \in L_{1}(\mu)_{+}$, one has

$$
H(U f \mid g) \leqq H(f \mid g)
$$

Proof. This is a trivial consequence of Theorem 1.1.

We now fix the measure space $(\Omega, \mathscr{A}, \mu)$. Furthermore, we assume that a measurable function $\alpha: \Omega \rightarrow\left[\alpha_{0}, \infty\right.$ ) (for some $\alpha_{0}>0$ ) is given. The $L_{1}$-norm in $L_{1}(\alpha \mu)$ will be denoted by $\|\cdot\|_{\alpha}$. We shall always assume the condition

$$
\exp (-\alpha) \in L_{1}(\mu)
$$

$[$ where $\exp (-\alpha)(w):=\exp (-\alpha(w))(w \in \Omega)]$. For $f \in L_{1}(\alpha \mu)_{+}$we define the negative entropy of $f$ by

$$
H(f):=\int f \ln f d \mu .
$$

We note that always $-\infty<H(f) \leqq \infty$, because of

$$
H(f)=H(f \mid \exp (-\alpha))-\|f\|_{\alpha} .
$$

1.6. Corollary. Let $(\alpha 1)$ be satisfied. Let $U: L_{1}(\mu) \rightarrow L_{1}(\mu)$ be a stochastic operator. Assume that $\exp (-\alpha)$ is a fixed point of $U$.

(a) Then $H(U f)+\|U f\|_{\alpha} \leqq H(f)+\|f\|_{\alpha}$ for all $f \in L_{1}(\alpha \mu)_{+}$such that $U f \in L_{1}(\alpha \mu)$.

(b) If $f \in L_{1}(\alpha \mu)_{+}$is such that $U f \in L_{1}(\alpha \mu)$ and $\|U f\|_{\alpha} \geqq\|f\|_{\alpha}$, then $H(U f) \leqq H(f)$ holds.

Proof. This follows from Corollary 1.5 and (1.14).

\section{Properties of $\boldsymbol{H}$-Decreasing Stochastic Operators}

In this section, we fix the measure space $(\Omega, \mathscr{A}, \mu)$. As in Sect. 1 , let $\alpha$ : $\Omega \rightarrow\left[\alpha_{0}, \infty\right.$ ) (for some $\alpha_{0}>0$ ) be a measurable function satisfying $(\alpha 1)$. 
We define the $\sigma$-algebra $\mathscr{A}_{\alpha}:=\left\{\alpha^{-1}(B) ; B \subset[0, \infty)\right.$ Borel set $\} \subset \mathscr{A}$ on $\Omega$. $\mu \mid \mathscr{A}_{\alpha}$ is $\sigma$-finite, since the sequence $A_{j}:=\{w \in \Omega ; \alpha(w) \leqq j\} \quad(j \in \mathbb{N})$ satisfies $A_{j} \in \mathscr{A}_{\alpha}, \Omega=\bigcup A_{j}, \mu\left(A_{j}\right) \leqq e^{j}\|\exp (-\alpha)\|<\infty$. For $f \in L_{1}(\mu)$ we define the signed measure $\mu_{f}$ on $\mathscr{A}_{\alpha}$ by $\mu_{f}(A):=\int_{A} f d \mu\left(A \in \mathscr{A}_{\alpha}\right)$. Then $\mu_{f}$ is absolutely continuous with respect to $\mu \mid \mathscr{A}_{\alpha}$, and the Radon-Nikodym theorem implies the existence of a unique $M_{\alpha} f \in L_{1}\left(\mu \mid \mathscr{A}_{\alpha}\right)$ such that

$$
\int_{A} M_{\alpha} f d \mu=\int_{A} f d \mu
$$

holds for all $A \in \mathscr{A}_{\alpha}$ (cf. [10, Theorem 6.9, p. 122 as well as the remarks on p. 124]). $M_{\alpha}$ is the operator which, to each $f \in L_{1}(\mu)$, assigns the conditional expectation with respect to $\mathscr{A}_{\alpha}$. The operator $M_{\alpha}: L_{1}(\mu) \rightarrow L_{1}\left(\mu \mid \mathscr{A}_{\alpha}\right)$ is stochastic. If $L_{1}\left(\mu \mid \mathscr{A}_{\alpha}\right)$ is canonically embedded in $L_{1}(\mu)$, and, accordingly, $M_{\alpha}$ is considered as an operator in $L_{1}(\mu)$, then $M_{\alpha}^{2}=M_{\alpha}$ holds.

2.1. Theorem. Let $(\alpha 1)$ be satisfied. Let $U: L_{1}(\mu) \rightarrow L_{1}(\mu)$ be a stochastic operator. Then the following statements are equivalent:

(a) $U\left(L_{1}(\alpha \mu)\right) \subset L_{1}(\alpha \mu)$, and $\|U f\|_{\alpha} \leqq\|f\|_{\alpha}, H(U f) \leqq H(f)$ for all $f \in L_{1}(\alpha \mu)_{+}$.

(b) $\exp (-\kappa \alpha)$ is a fixed point of $U$ for all $\kappa \geqq 1$ (or equivalently, for all $\kappa>0$ such that $\left.\exp (-\kappa \alpha) \in L_{1}(\mu)\right)$.

(c) $U f=f$ for all $f \in L_{1}\left(\mu \mid \mathscr{A}_{\alpha}\right.$ ) (or expressed differently, $U M_{\alpha}=M_{\alpha}$ ).

(d) $U M_{\alpha}=M_{\alpha}=M_{\alpha} U$.

(e) $U\left(L_{1}(\alpha \mu)\right) \subset L_{1}(\alpha \mu),\|U f\|_{\alpha}=\|f\|_{\alpha}$ for all $f \in L_{1}(\alpha \mu)_{+}$, and $\exp (-\alpha)$ is a fixed point of $U$.

We note that, if $\kappa^{\prime}>0$ is such that $\exp \left(-\kappa^{\prime} \alpha\right) \in L_{1}(\mu)$, then $\exp (-\kappa \alpha)$ $\in L_{1}(\alpha \mu)$ for all $\kappa>\kappa^{\prime}$. Therefore $(\alpha 1)$ implies $\exp (-\kappa a) \in L_{1}(\alpha \mu)$ for all $\kappa>1$.

For the proof of Theorem 2.1 we need some preparation.

2.2. Lemma ("Lemma of Gibbs"). Let $\exp (-\alpha) \in L_{1}(\alpha \mu)$, and assume that $f \in L_{1}(\alpha \mu)_{+}$satisfies

$$
\|f\| \geqq\|\exp (-\alpha)\|, \quad\|f\|_{\alpha} \leqq\|\exp (-\alpha)\|_{\alpha}, \quad H(f) \leqq H(\exp (-\alpha)) .
$$

Then $f=\exp (-\alpha)$.

Proof (compare [2, p. 25], [8, p. 549]). The assumptions imply

$$
\begin{aligned}
& 0 \leqq\|f\|-\|\exp (-\alpha)\| \leqq H(f \mid \exp (-\alpha))=H(f)+\|f\|_{\alpha} \leqq H(\exp (-\alpha))+\|\exp (-\alpha)\|_{\alpha} \\
& =H(\exp (-\alpha) \mid \exp (-\alpha))=0 \text {, }
\end{aligned}
$$

thus $H(f \mid \exp (-\alpha))=\|f\|-\|\exp (-\alpha)\|$, which implies $f=\exp (-\alpha)$.

2.3. Lemma. Let $(\alpha 1)$ be satisfied. Then the set $\{\exp (-\kappa \alpha) ; \kappa \geqq 1\}$ is total in $L_{1}\left(\mu \mid \mathscr{A}_{\alpha}\right)$ (i.e. the linear span of $\{\ldots\}$ is dense).

Proof. On the $\sigma$-algebra $\mathscr{A}^{\prime}$ of Borel sets of $[0, \infty)$ we define the image $\mu^{\prime}$ of $\mu$ under $\alpha, \mu^{\prime}(B):=\mu\left(\alpha^{-1}(B)\right)\left(B \in \mathscr{A}^{\prime}\right)$. Obviously $\mu^{\prime}(B)<\infty$ for each compact set 
$B \subset[0, \infty)$. The operator $J: L_{1}\left(\mu^{\prime}\right) \rightarrow L_{1}\left(\mu \mid \mathscr{A}_{\alpha}\right), J f^{\prime}:=f^{\prime} \circ \alpha\left(f^{\prime} \in L_{1}\left(\mu^{\prime}\right)\right)$, defines an isometric isomorphism (cf. [9, Chap. 15.2, Proposition 1, p. 318]).

For $\kappa \geqq 1$ we define $f_{\kappa}^{\prime}:[0, \infty) \rightarrow \mathbb{R}, f_{\kappa}^{\prime}(t):=e^{-\kappa t}$. Then $f_{\kappa}^{\prime} \circ \alpha=\exp (-\kappa \alpha)$, and therefore $f_{\kappa}^{\prime} \in L_{1}\left(\mu^{\prime}\right)$. Now, the assertion is equivalent to the statement that $\left\{f_{\kappa}^{\prime}\right.$; $\kappa \geqq 1\}$ is total in $L_{1}\left(\mu^{\prime}\right)$; we are going to prove the latter statement.

Because of $f_{1}^{\prime} \in L_{1}\left(\mu^{\prime}\right)_{+}, \mu^{\prime \prime}:=f_{1}^{\prime} \mu^{\prime}$ is an integrable measure on $[0, \infty)$. The operator $J^{\prime}: L_{1}\left(\mu^{\prime}\right) \rightarrow L_{1}\left(\mu^{\prime \prime}\right), J^{\prime} f^{\prime}(t):=e^{t} f^{\prime}(t)(t \geqq 0)$, is an isometric isomorphism, and we have $J^{\prime} f_{\kappa}^{\prime}=f_{\kappa}^{\prime \prime}$, where $f_{\kappa}^{\prime \prime}(t):=e^{-(\kappa-1) t}(t \geqq 0)$, for all $\kappa \geqq 1$. So it remains to show that $A:=\left[f_{\kappa}^{\prime \prime} ; \kappa \geqq 1\right]$ (=linear subspace of $L_{1}\left(\mu^{\prime \prime}\right)$ generated by $\left\{f_{\kappa}^{\prime \prime}\right.$; $\kappa \geqq 1\})$ is dense in $L_{1}\left(\mu^{\prime \prime}\right)$. Obviously, $A$ is a subalgebra of $C([0, \infty])(\cong$ space of continuous functions on $[0, \infty)$ converging at $\infty)$, and $A$ separates the points of $[0, \infty]$ and contains the function $1=f_{1}^{\prime \prime}$. Therefore, $A$ is $\|\cdot\|_{\infty}$-dense in $C([0, \infty])$, by the Stone-Weierstrass theorem ([1, Chap. X, Sect. 4.2, Theorem 3 , p. 36]). Because of $\mu^{\prime \prime}([0, \infty])<\infty,\left(C([0, \infty]),\|\cdot\|_{\infty}\right) \hookrightarrow\left(L_{1}\left(\mu^{\prime \prime}\right),\|\cdot\|\right)$ exists and is continuous, and $C([0, \infty])$ is dense in $L_{1}\left(\mu^{\prime \prime}\right)$. This shows that $A$ is dense in $L_{1}\left(\mu^{\prime \prime}\right)$.

2.4. Lemma. Let $(\alpha 1)$ be satisfied. If $f \in L_{1}(\alpha \mu)$, then we have $M_{\alpha} f \in L_{1}(\alpha \mu)$, $M_{\alpha}(\alpha f)=\alpha M_{\alpha} f$. Further, if $f \in L_{1}(\mu)_{+}$and $M_{\alpha} f \in L_{1}(\alpha \mu)$, then $f \in L_{1}(\alpha \mu)$.

Proof. The bounded $\mathscr{A}_{\alpha}$-measurable function $\alpha^{-1}$ can be approximated uniformly by a sequence $\left(\xi_{n}\right)$ of $\mathscr{A}_{\alpha}$-simple functions. The obvious equalities $\xi_{n} M_{\alpha} f$ $=M_{\alpha}\left(\xi_{n} f\right)(n \in \mathbb{N})$ imply $\alpha^{-1} M_{\alpha} f=M_{\alpha}\left(\alpha^{-1} f\right)\left(f \in L_{1}(\mu)\right)$. If $f \in L_{1}(\alpha \mu)$, then $M_{\alpha} f$ $=M_{\alpha}\left(\alpha^{-1} \alpha f\right)=\alpha^{-1} M_{\alpha}(\alpha f)$ implies the first assertion. To show the second assertion, let $\chi_{n}$ be the indicator function of the set $\{w \in \Omega ; \alpha(w) \leqq n\} \quad(n \in \mathbb{N})$. Then $M_{\alpha}\left(\chi_{n} f\right)=\chi_{n} M_{\alpha} f, \quad \int \chi_{n}(\alpha f) d \mu=\int \alpha M_{\alpha}\left(\chi_{n} f\right) d \mu=\int \chi_{n} \alpha M_{\alpha} f d \mu \rightarrow \int \alpha M_{\alpha} f d \mu$, and $\alpha f \in L_{1}(\mu)$ follows from the monotone convergence theorem.

Proof of Theorem 2.1. (a) $\Rightarrow$ (b). Let $\kappa^{\prime}>0$ be such that $\exp \left(-\kappa^{\prime} \alpha\right) \in L_{1}(\mu)$, and let $\kappa>\kappa^{\prime}$. Then $\exp (-\kappa \alpha) \in L_{1}(\alpha \mu)$. If, in Lemma 2.2, $\alpha$ is replaced by $\kappa \alpha$, then for $f:=U \exp (-\kappa \alpha)$ the assumptions are satisfied, and $f=\exp (-\kappa \alpha)$ follows. From $\exp (-\kappa \alpha) \rightarrow \exp \left(-\kappa^{\prime} \alpha\right)\left(\kappa \rightarrow \kappa^{\prime}+\right)$ we obtain $U \exp \left(-\kappa^{\prime} \alpha\right)=\exp \left(-\kappa^{\prime} \alpha\right)$.

(b) $\Rightarrow(\mathrm{c})$. From $U \exp (-\kappa \alpha)=\exp (-\kappa \alpha)(\kappa \geqq 1)$ and Lemma 2.3 we obtain $U f=f$ for all $f \in L_{1}\left(\mu \mid \mathscr{A}_{\alpha}\right)$.

(c) $\Rightarrow$ (d). First we show that $f \in L_{1}(\mu)_{+}, A \in \mathscr{A}_{\alpha}, f \mid \mathfrak{l} A=0$ implies $U f \mid \mathfrak{\complement} A$ $=0$. If $\mu(A)<\infty$, then $\chi_{A} \in L_{1}\left(\mu \mid \mathscr{A}_{\alpha}\right), f=\lim \left(f \wedge j \chi_{A}\right), U f=\lim U\left(f \wedge j \chi_{A}\right)$, and from $0 \leqq U\left(f \wedge j \chi_{A}\right) \leqq U\left(j \chi_{A}\right)=j \chi_{A}(j \in \mathbb{N})$ we obtain $U f \mid \mathfrak{l} A=0$. In the case $\mu(A)=\infty$ there exists an increasing sequence $\left(A_{j}\right)$ in $\mathscr{A}_{\alpha}, \bigcup A_{j}=A, \mu\left(A_{j}\right)<\infty$ $(j \in \mathbb{N})$. We have $f=\lim \chi_{A_{j}} f$, and from $U\left(\chi_{A_{j}} f\right) \mid \mathcal{\complement} A=0$ and $U f=\lim U\left(\chi_{A_{f}} f\right)$ we obtain $U f \mid \mathfrak{l} A=0$.

Now, let $f \in L_{1}(\mu)$. If $A \in \mathscr{A}_{\alpha}$, then the fact shown in the preceding paragraph implies $\chi_{A} U\left(\chi_{A} f\right)=U\left(\chi_{A} f\right),\left(1-\chi_{A}\right) U\left(\left(1-\chi_{A}\right) f\right)=U\left(\left(1-\chi_{A}\right) f\right)$, which in turn implies $\chi_{A} U f=\chi_{A} U\left(\chi_{A} f\right)+\chi_{A} U\left(\left(1-\chi_{A}\right) f\right)=U\left(\chi_{A} f\right)$,

$$
\int_{A} U f d \mu=\int_{\Omega} U\left(\chi_{A} f\right) d \mu=\int_{\Omega} \chi_{A} f d \mu=\int_{A} f d \mu .
$$

Since this equality holds for all $A \in \mathscr{A}_{\alpha}$, the definition of $M_{\alpha}$ implies $M_{\alpha} f$ $=M_{\alpha} U f$. This shows $M_{\alpha}=M_{\alpha} U$. 
(d) $\Rightarrow$ (e). If $f \in L_{1}(\alpha \mu)$, then $M_{\alpha} U f^{ \pm}=M_{\alpha} f^{ \pm} \in L_{1}(\alpha \mu)$ implies $U f^{ \pm} \in L_{1}(\alpha \mu)$, by Lemma 2.4. For $f \in L_{1}(\alpha \mu)_{+}$, Lemma 2.4 implies

$$
\|U f\|_{\alpha}=\|\alpha U f\|=\left\|M_{\alpha}(\alpha U f)\right\|=\left\|\alpha M_{\alpha} U f\right\|=\left\|\alpha M_{\alpha} f\right\|=\left\|M_{\alpha}(\alpha f)\right\|=\|\alpha f\|=\|f\|_{\alpha} .
$$

From $\exp (-\alpha) \in L_{1}\left(\mu \mid \mathscr{A}_{\alpha}\right)$ we obtain $U \exp (-\alpha)=U M_{\alpha} \exp (-\alpha)=M_{\alpha} \exp (-\alpha)$ $=\exp (-\alpha)$.

(e) $\Rightarrow(a)$. This was proved in Corollary 1.6(b).

\subsection{Proposition. Assume that}

$$
\exp (-\kappa \alpha) \in L_{1}(\mu) \quad \text { for all } \kappa>0
$$

holds. Let $U: L_{1}(\mu) \rightarrow L_{1}(\mu)$ be a stochastic operator satisfying one (and therefore all) of the conditions of Theorem 2.1. Then $\|U f\|_{\infty} \leqq\|f\|_{\infty}$ holds for all $f \in L_{1}(\mu) \cap L_{\infty}(\mu)$.

Proof. Let $f \in L_{1}(\mu) \cap L_{\infty}(\mu)$, without restriction $f \geqq 0$ and $\|f\|_{\infty}=1$. Let $f_{k}:=f$ $\wedge \exp (-\alpha / k)(k \in \mathbb{N})$. Then $f_{k} \nearrow f(k \rightarrow \infty)$ in $L_{1}(\mu)$. This implies $U f_{k} \nearrow U f$ in $L_{1}(\mu)$, in particular $U f_{k} \rightarrow U f \mu$-almost everywhere. From (b) of Theorem 2.1 we have $U f_{k} \leqq U \exp (-\alpha / k)=\exp (-\alpha / k) \leqq 1,\left\|U f_{k}\right\|_{\infty} \leqq 1 \quad(k \in \mathbb{N})$. This implies $\|U f\|_{\infty} \leqq 1$.

\section{References}

1. Bourbaki, N.: Topologie générale. Chaps. 5-10. Paris: Hermann 1974

2. Carleman, T.: Problèmes mathématiques dans la théorie cinétique des gaz. Uppsala: Almquist \& Wiksells 1957

3. Dunford, N., Schwartz, J.T.: Linear operators, Part I: General theory. New York: Interscience Publishers 1958

4. Hille, E., Phillips, R.S.: Functional analysis and semi-groups. Providence, R.I.: Am. Math. Soc. Coll. Publ. 1957

5. Kullback, S.: Information theory and statistics. New York: Wiley 1959

6. Lindblad, G.: Commun. Math. Phys. 40, 147-151 (1975)

7. Moreau, M.: J. Math. Phys. 19, 2494-2498 (1978)

8. Morgenstern, D.: J. Rational Mech. Anal. 4, 533-555 (1955)

9. Royden, H.L.: Real analysis. New York: Macmillan Publ. Co. 1968

10. Rudin, W.: Real and complex analysis. New York: McGraw-Hill 1966

11. Schaefer, H.H.: Banach lattices and positive operators. Berlin, Heidelberg, New York: Springer 1974

12. Umegaki, H.: Kodai Math. Sem. Rep. 14, 59-85 (1962)

13. Voigt, J.: The $H$-theorem for Boltzmann type equations. J. Reine Angew. Math. (to appear)

14. Voigt, J.: Functional analytic treatment of the initial boundary value problem for collisionless gases. Habilitationsschrift, Universität München 1981

Communicated by E. Lieb

Received February 17, 1981 